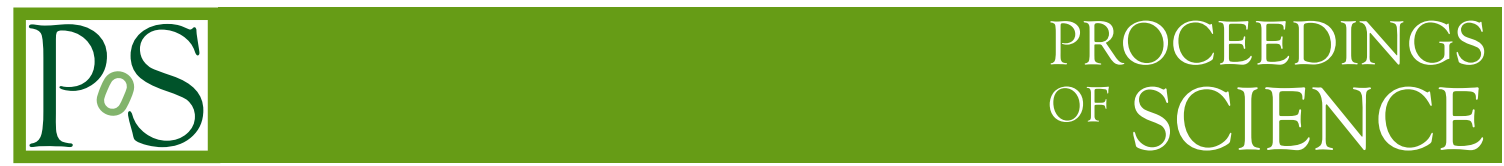

\title{
FCNC and EFT interpretations in top quark events in CMS
}

\author{
Samuel May for the CMS Collaboration ${ }^{a, *}$ \\ ${ }^{a}$ University of California, San Diego \\ 9500 Gilman Dr., La Jolla, CA, 92093, United States of America \\ E-mail: sjmay@ucsd.edu
}

\begin{abstract}
Top quark production can probe physics beyond the standard model (SM) in different ways. The Effective Field Theory (EFT) framework allows searching for beyond the SM (BSM) effects in a model independent way. CMS experiment is pioneering EFT measurements that move towards using full potential of the data in the most global way possible. Searches for flavour-changing neutral currents (FCNC) and anomalous top quark interactions are also being pursued in CMS which are complementary to the EFT approach. This talk reviews the current limits on FCNC searches in the top sector, and EFT interpretations.
\end{abstract}

40th International Conference on High Energy physics - ICHEP2020

July 28 - August 6, 2020

Prague, Czech Republic (virtual meeting)

\footnotetext{
${ }^{*}$ Speaker
} 


\section{Introduction}

The standard model of particle physics does not provide a complete description of the universe and has a variety of shortcomings, including its lack of explanation for dark matter \& dark energy, its inability to explain the observed matter-antimatter asymmetry, and the strong $\mathrm{CP}$ problem. These shortcomings motivate searches for new physics phenomena at the LHC; however, no clear signs of BSM physics have been observed yet. This lack of discovery motivates the notion that new physics may exist at masses which are beyond the energy reach of the LHC. In such scenarios, new physics might still present itself in the form of various anomalous interactions at rates which are measurable at the LHC. Any deviations from SM predictions can be described in a modelindependent way within the EFT framework, which parametrizes these deviations in terms of coefficients of dimension-six operators added to the SM Lagrangian (SMEFT) [2, 3].

In top quark events, there are two primary methods of searching for such effects of new physics. First, through measurements of SM processes involving top quarks with an interpretation of the results in terms of constraints of coefficients on dimension-six EFT operators. Second, through searches for explicitly forbidden processes in the SM, like FCNCs.

In the SM, FCNCs can only proceed through higher-order diagrams whose rates are heavily suppressed by the Glashow-Iliopoulos-Maiani (GIM) mechanism; they are forbidden at tree-level. The predicted SM branching fractions are on the order of $10^{-17}-10^{-12}$ [4], making them undetectable at the LHC - any observation of FCNC interactions would be an unambiguous sign of new physics. A number of BSM models predict enhanced branching fractions of top quark FCNC decays, including composite Higgs models, warped extra dimensions models, quark-singlet models [5], and two-Higgs double models (2HDM) either with or without explicit flavor violation [6-8]. Two particularly compelling scenarios are those of a composite Higgs boson or warped extra dimensions, where the branching fraction for $\mathrm{t} \rightarrow \mathrm{Hc}(\mathrm{t} \rightarrow \mathrm{Zc})$ could be as high as $10^{-4}\left(10^{-5}\right)$, with rates for the other potential FCNC mediators ( $\mathrm{g}, \gamma$ ) well below LHC sensitivity [4].

\section{Probing new physics in associated top quark production}

Processes involving the production of one or more top quarks are sensitive to a variety of dimension-six operators, while typical LHC studies are only sensitive to a few of these operators. A novel approach [9] targets a variety of associated top quark production modes and is designed with the explicit goal of minimizing constraints on the various Wilson coefficients (WCs) of the dimension-six operators, setting constraints on sixteen total WCs. The study utilizes $41.5 \mathrm{fb}^{-1}$ of $13 \mathrm{TeV}$ CMS [1] data and considers the production of one or more top quarks in association with a $\mathrm{W}$ boson, $\mathrm{Z}$ boson, or Higgs boson $(\mathrm{H})$. Rather than trying to isolate specific physics processes, this study categorizes events based on detector-level observables, namely the number of charged leptons, hadronic jets, and hadronic jets tagged as originating from a b quark ("b-jets"). The novel approach in this study lies in the fact that the yields for each process with prompt leptons are parametrized in terms of the WCs of all operators simultaneously.

The categorization of events according to lepton flavor, lepton charge multiplicity, and jet multiplicity results in 35 statistically independent regions. Constraints on WCs are then determined through a binned likelihood fit in which the event yields are a function of both the WCs and a 
set of nuisance parameters which take into account the effects of systematic uncertainties. While a simultaneous fit of all $16 \mathrm{WCs}$ can be performed, in principle, this is very time-consuming in practice; instead, constraints are obtained in two ways: first, one WC is constrained while the other 15 WCs are treated as unconstrained nuisance parameters ("profiled") and second, one WC is constrained while the other $15 \mathrm{WCs}$ are fixed to their SM value of zero. The results of these fits are shown in Fig. 1.
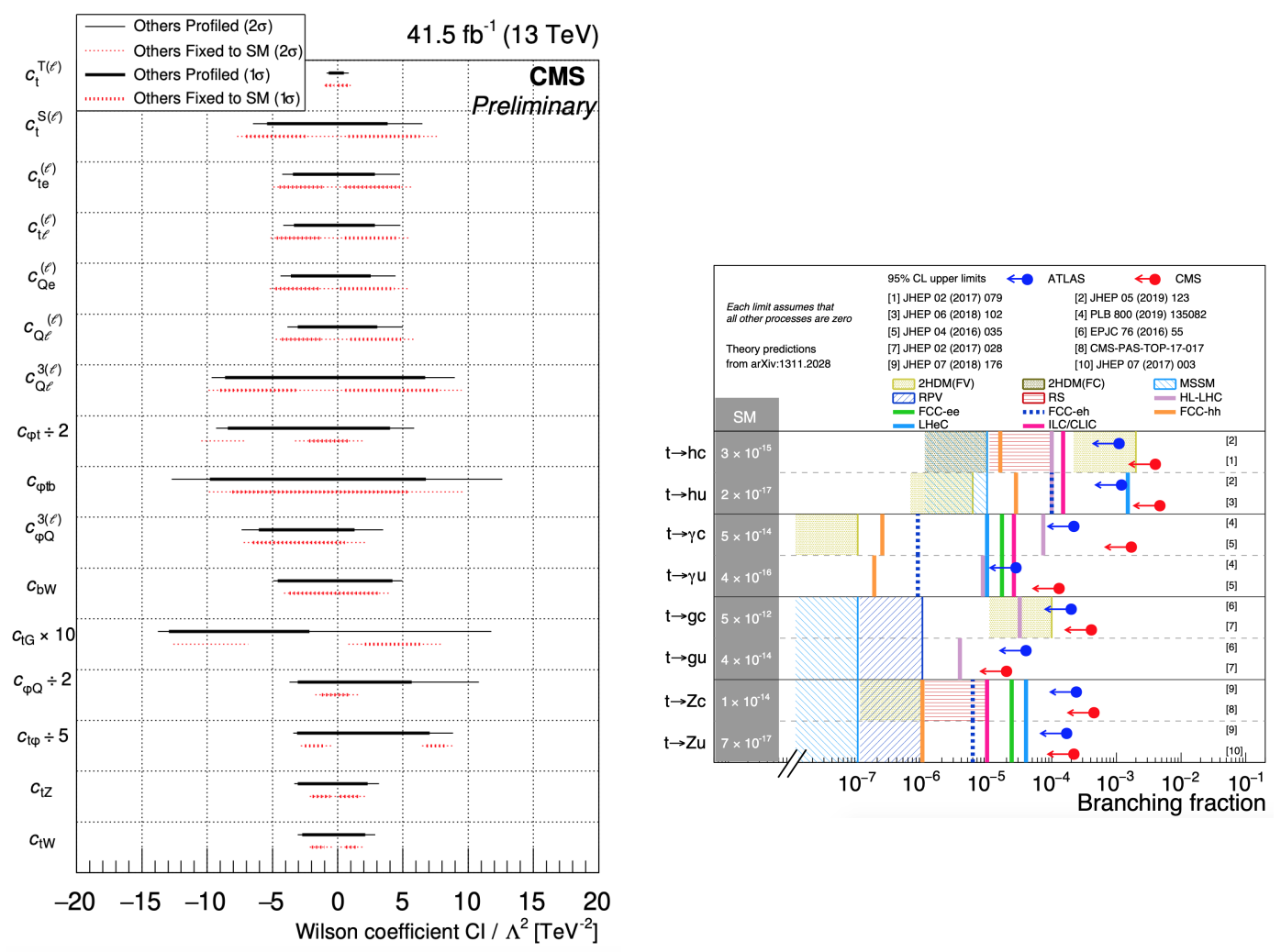

Figure 1: Left: Observed confidence intervals placed on WCs, shown for the fitting scenario in which all other WCs are profiled (black) and the fitting scenario in which all other WCs are fixed to their SM value (red). Taken from [9].

Right: Current exclusion limits and projections for limits possible with future colliders on the top quark FCNC branching fractions. Experimental constraints are shown along with ranges of predicted branching fractions for a variety of BSM models. Updated version of Fig. 6.4 from [14].

\section{EFT constraints from $\mathrm{t} \bar{t}, \mathrm{tW}, \mathrm{t} \overline{\mathrm{Z}}$, and four top measurements}

Additional constraints on WCs of dimension-six EFT operators related to top quark production were placed by studies measuring production of a top quark-antiquark pair in association with a $\mathrm{Z}$ boson [10], searching for the production of four top quarks [11], searching for new physics in dilepton final states of $\mathrm{t} \overline{\mathrm{t}}$ and $\mathrm{tW}$ events [12], and in measurements of the top quark polarization and $\mathrm{t} \overline{\mathrm{t}}$ spin correlations [13]. 
Measurements of the cross section, both inclusive and differential, for the production of a top quark-antiquark pair in association with a $\mathrm{Z}$ boson were performed [10] with $77.5 \mathrm{fb}^{-1}$ of $13 \mathrm{TeV}$ CMS data, considering final states with three or four leptons. In the context of SMEFT, the t⿱亠乂ZZ process is especially sensitive to the following WCs: $c_{\mathrm{tZ}}, c_{\mathrm{tZ}}^{[I]}, c_{\phi t}$, and $c_{\phi Q}^{-}$. The predicted event yields for scenarios of non-zero WCs are obtained through simulations of the signal sample binned in a fine grid in the relevant WC parameter space (all other WCs are assumed to be SM-like). A binned likelihood function is then constructed as a function of the predicted yields and uncertainties which is then used to set constraints on the values of WCs, found to be in agreement with the SM predictions [10].

The search for the production of four top quarks [11] uses $35.8 \mathrm{fb}^{-1}$ of $13 \mathrm{TeV}$ CMS data and targets single lepton and opposite-sign dilepton final states. A variety of BSM models result in a modified cross section for four top production. Under the assumption that new physics couples to the left-handed doublet and right-handed up-type quark singlet of the third generation, only four EFT operators $\left(O_{\mathrm{tt}}^{1}, O_{Q Q}^{1}, O_{Q \mathrm{t}}^{1}, O_{Q \mathrm{t}}^{8}\right)$ will affect the four top production cross section.Event selection is performed through the use of boosted decision trees (BDTs) that are trained with variables describing the event-level kinematics, with the four top production process as signal and relevant SM processes as background. The observed constraints on the WCs of these operators are found to be in agreement with the SM: $[-2.1,2.0]\left(O_{\mathrm{tt}}^{1}\right),[-2.2,2.0]\left(O_{Q Q}^{1}\right),[-3.5,3.5]\left(O_{Q \mathrm{t}}^{1}\right)$, and $[-7.9,6.6]$ $\left(O_{Q t}^{8}\right)$, in units of $\mathrm{TeV}^{-2}$.

The search for new physics in dilepton final states of t⿱t and tW [12] uses $35.9 \mathrm{fb}^{-1}$ of $13 \mathrm{TeV}$ CMS data and specifically exploits the sensitivity of the tW process to new physics, the first paper to do so. In the EFT framework the following WCs are especially sensitive to tW production: $C_{\phi \mathrm{q}}^{(3)}$, $C_{\mathrm{tW}}, C_{\mathrm{tG}}$, and $C_{\mathrm{u}(\mathrm{c}) \mathrm{G}} . C_{\phi \mathrm{q}}^{(3)}$ and $C_{\mathrm{tW}}$ correspond to anomalous Wtb couplings, $C_{\mathrm{tG}}$ corresponds to a chromomagnetic dipole moment, and $C_{\mathrm{u}(\mathrm{c}) \mathrm{G}}$ correspond to FCNC interactions. The WC of the triple gluon strength operator, $C_{\mathrm{G}}$ is also constrained in this study, though this is sensitive only to $\bar{t} \bar{t}$ production, not $\mathrm{tW}$ production. A deep neural network (DNN) trained to separate between the $\bar{t} \bar{t}$ and $\mathrm{tW}$ processes is used to isolate regions of data which are enriched in tW events. Event yields for $\mathrm{t} \overline{\mathrm{t}}$ and $\mathrm{tW}$ are parametrized as a function of WC values and constraints are extracted through a binned likelihood fit, with the results in agreement with the SM expectations [12].

Measurements of the top quark polarization and $\bar{t}$ spin correlations [13] are performed with $35.9 \mathrm{fb}^{-1}$ of $13 \mathrm{TeV}$ CMS data. The study examines final states with opposite-sign dilepton pairs and additional jets which are tagged as originating from $a b$ quark. Constraints are placed on each of the independent coefficients relating to spin of the $t \bar{t}$ production density matrix. A variety of WCs, including the chromomagnetic $\left(C_{\mathrm{tG}}\right)$ and chromoelectric $\left(C_{\mathrm{tG}}^{I}\right)$ dipole moments, can then be expressed as a function of the density matrix coefficients. The observed measurements of the chromomagnetic and chromomagnetic dipole moments, as well as a variety of other anomalous couplings, are found to be in agreement with the SM [13].

\section{Searches for top quark FCNC decays}

Searches for top quark FCNC decays are performed in various channels at CMS, including Z, $\mathrm{H}, g$ and $\gamma$. Current constraints from both ATLAS and CMS are summarized in Fig. 1, with this section focusing on the CMS results in the $\mathrm{t} \rightarrow \mathrm{Hq}$ and $\mathrm{t} \rightarrow \mathrm{Zq}$ channels. 
A search for top FCNC interactions mediated by a Z boson [15] was performed with $36 \mathrm{fb}^{-1}$ of $13 \mathrm{TeV}$ CMS data, targeting final states with exactly three leptons. The three leptons are required to include an opposite-sign same-flavor (OSSF) pair, expected from the leptonic decay of the Z boson. The study considers both single top and $\mathrm{t} \overline{\mathrm{t}}$ production modes, with BDTs trained to target each production mode. The distributions of BDT discriminants are then used to perform a likelihood fit and extract constraints on the FCNC branching fractions. The observed (expected) limits on $\mathcal{B}(\mathrm{t} \rightarrow \mathrm{Zc})$ and $\mathcal{B}(\mathrm{t} \rightarrow \mathrm{Zu})$ are $4.5 \times 10^{-4}\left(3.7 \times 10^{-4}\right)$ and $2.4 \times 10^{-4}\left(1.5 \times 10^{-4}\right)$, respectively.

A search for top FCNC interactions mediated by a Higgs boson [16] was also performed with $36 \mathrm{fb}^{-1}$ of $13 \mathrm{TeV}$ CMS data, targeting the $\mathrm{b} \bar{b}$ decay mode of the $\mathrm{H}$ and both single top and $\bar{t} \mathrm{t}$ signal production modes. A BDT is trained to discriminate between signal processes and the dominant SM backgrounds, primarily tt plus additional hadronic jets. Events are then categorized according to the number of hadronic jets and number of b-jets; constraints on the FCNC branching fractions are extracted with a likelihood fit over the categories. The observed (expected) limits on $\mathcal{B}(\mathrm{t} \rightarrow \mathrm{Hc})$ and $\mathcal{B}(\mathrm{t} \rightarrow \mathrm{Hu})$ are $4.7 \times 10^{-4}\left(4.4 \times 10^{-4}\right)$ and $4.7 \times 10^{-4}\left(3.4 \times 10^{-4}\right)$, respectively.

\section{Conclusions \& future prospects}

Despite strong motivations for the existence of physics beyond the SM, the LHC has not yet delivered any clear indication of new physics phenomena. One possibility for the lack of discovery thus far is the notion that new physics may exist, but at masses beyond the energy reach of the LHC. Even in this case, LHC experiments could still be sensitive to the effects of new physics, which could manifest themselves as anomalous couplings of the top quark or forbidden interactions, like FCNC decays. The EFT framework provides a model-independent way to interpret these results, as detailed in the results $[9-13,15,16]$ described in these proceedings. Though these results are in agreement with the SM expectations, providing no hint of new physics, future developments will help to further clarify the landscape. As Fig. 1 shows, expected exclusion limits on top FCNC branching fractions are expected to improve drastically with the HL-LHC dataset, often by more than an order of magnitude. Many scenarios of BSM physics result in enhanced branching fractions for top FCNC decays which are not excluded by current limits but would be observable at the HL-LHC [14].

\section{References}

[1] S. Chatrchyan et al. [CMS], "The CMS Experiment at the CERN LHC," JINST 3, S08004 (2008) doi:10.1088/1748-0221/3/08/S08004

[2] W. Buchmuller and D. Wyler, "Effective Lagrangian Analysis of New Interactions and Flavor Conservation,” Nucl. Phys. B 268, 621-653 (1986) doi:10.1016/0550-3213(86)90262-2

[3] B. Grzadkowski, M. Iskrzynski, M. Misiak and J. Rosiek, "Dimension-Six Terms in the Standard Model Lagrangian," JHEP 10, 085 (2010) doi:10.1007/JHEP10(2010)085 [arXiv: 1008.4884 [hep-ph]].

[4] K. Agashe et al. [Top Quark Working Group], "Working Group Report: Top Quark," [arXiv:1311.2028 [hep-ph]]. 
[5] J. A. Aguilar-Saavedra, "Effects of mixing with quark singlets," Phys. Rev. D 67, 035003 (2003) [erratum: Phys. Rev. D 69, 099901 (2004)] doi:10.1103/PhysRevD.69.099901 [arXiv:hep$\mathrm{ph} / 0210112$ [hep-ph]].

[6] S. Bejar, J. Guasch and J. Sola, "Loop induced flavor changing neutral decays of the top quark in a general two Higgs doublet model," Nucl. Phys. B 600, 21-38 (2001) doi:10.1016/S05503213(01)00044-X [arXiv:hep-ph/0011091 [hep-ph]].

[7] J. Guasch and J. Sola, "FCNC top quark decays: A Door to SUSY physics in high luminosity colliders?,” Nucl. Phys. B 562, 3-28 (1999) doi:10.1016/S0550-3213(99)00579-9 [arXiv:hepph/9906268 [hep-ph]].

[8] J. Cao, C. Han, L. Wu, J. M. Yang and M. Zhang, "SUSY induced top quark FCNC decay $t \rightarrow c h$ after Run I of LHC," Eur. Phys. J. C 74, no.9, 3058 (2014) doi:10.1140/epjc/s10052014-3058-1 [arXiv:1404.1241 [hep-ph]].

[9] CMS Collaboration, "Using associated top quark production to probe for new physics within the framework of effective field theory," CMS-PAS-TOP-19-001, https://cds.cern.ch/record/2725399

[10] A. M. Sirunyan et al. [CMS], "Measurement of top quark pair production in association with a $\mathrm{Z}$ boson in proton-proton collisions at $\sqrt{s}=13 \mathrm{TeV}$," JHEP 03, 056 (2020) doi:10.1007/JHEP03(2020)056 [arXiv:1907.11270 [hep-ex]].

[11] A. M. Sirunyan et al. [CMS], "Search for the production of four top quarks in the single-lepton and opposite-sign dilepton final states in proton-proton collisions at $\sqrt{s}=13 \mathrm{TeV}$," JHEP 11, 082 (2019) doi:10.1007/JHEP11(2019)082 [arXiv:1906.02805 [hep-ex]].

[12] A. M. Sirunyan et al. [CMS], "Search for new physics in top quark production in dilepton final states in proton-proton collisions at $\sqrt{s}=13$ TeV," Eur. Phys. J. C 79, no.11, 886 (2019) doi:10.1140/epjc/s10052-019-7387-y [arXiv:1903.11144 [hep-ex]].

[13] A. M. Sirunyan et al. [CMS], "Measurement of the top quark polarization and t⿱亠䒑 spin correlations using dilepton final states in proton-proton collisions at $\sqrt{s}=13 \mathrm{TeV}$," Phys. Rev. D 100, no.7, 072002 (2019) doi:10.1103/PhysRevD.100.072002 [arXiv:1907.03729 [hep-ex]].

[14] A. Abada et al. [FCC], "FCC Physics Opportunities: Future Circular Collider Conceptual Design Report Volume 1,” Eur. Phys. J. C 79, no.6, 474 (2019) doi:10.1140/epjc/s10052-0196904-3

[15] CMS Collaboration, "Search for flavour changing neutral currents in top quark production and decays with three-lepton final state using the data collected at sqrt(s) $=13 \mathrm{TeV}$," CMS-PASTOP-17-017, https://cds.cern.ch/record/2292045

[16] A. M. Sirunyan et al. [CMS], "Search for the flavor-changing neutral current interactions of the top quark and the Higgs boson which decays into a pair of b quarks at $\sqrt{s}=13 \mathrm{TeV}$," JHEP 06, 102 (2018) doi:10.1007/JHEP06(2018)102 [arXiv:1712.02399 [hep-ex]]. 